\title{
LETTERS
}

doi:10.1017/S1041610212000385

\section{The CAMCOG for patients with Alzheimer's disease with low levels of education}

We would like to congratulate Aprahamian et al. (2011) for their initiative to investigate the psychometric properties of the Cambridge Cognition Examination (CAMCOG) in assessing the cognitive status of the Brazilian elderly. This instrument is very frequently used in Brazil, but its measure characteristics have not yet been fully determined. Of course, cross-cultural adaptation and validation are rarely a simple procedure, often demanding a research program to completely adapt the tool to the specific language, culture, and selected population. In our opinion, the paper "Can the CAMCOG be a good cognitive test for patients with Alzheimer's disease with low levels of education?" (Aprahamian et al., 2011) is welcome as another trial by Brazilian scientists in this ongoing process. Notwithstanding, we believe that some comments about the methodological aspects of the paper are in order.

Firstly, individuals rated with a Clinical Dementia Rating (CDR) of $0.5,1.0$, or 2.0 were classified as having mild or moderate Alzheimer's disease (AD). It was correct to exclude severe $\mathrm{AD}$ from the study protocol, since the CAMCOG was not designed to diagnose $\mathrm{AD}$ in an advanced stage. However, it was disturbing that the authors have classified patients with mild cognitive impairment (MCI; CDR $=0.5)$ as having mild AD. The issue here about what MCI means in terms of clinical and pathological evolution remains unresolved. It is widely known within the scientific community that there is a "growing consensus in the field that there is a phase of $\mathrm{AD}$ when individuals experience a gradually progressive cognitive decline, that results from the accumulation of $\mathrm{AD}$ pathology in the brain" (Albert et al., 2011), and that, until the present moment, patients are diagnosed with $\mathrm{AD}$ "when the cognitive impairment is sufficiently great, such that there is interference with daily function" (Albert et al., 2011). On the basis of recent evidence, these authors proposed to look upon this as a process in which MCI may be interpreted "as a subset of the many causes of cognitive impairment that are not dementia (CIND), including impairments resulting from head trauma, substance abuse, or metabolic disturbance" (Albert et al., 2011). Therefore, from the point of view of scientific evidence, it is not incorrect to classify as having $\mathrm{AD}$, some of the individuals diagnosed as having MCI, although in order to do this it is necessary to determine the individual status relative to biological markers of beta-amyloid protein deposition and neuronal injury, associated with AD (Albert et al., 2011). In their work, Aprahamian et al. (2011) considered all 37 individuals classified as $\mathrm{CDR}=0.5$ as suffering from AD. This was almost a third of their total AD sample (113) classified by a questionable criterion, given that there is no determination of biological markers in their study protocol.

Secondly, the authors' statement that "the CAMCOG in its original and revised versions are validated in Brazil" (Aprahamian et al., 2011) must be put in perspective. To this end, we draw on the well-established concept of equivalences, as developed by Herdman et al. (1998). To these authors, the adaptation to a new reality of an assessment instrument created in a different cultural background implies a process aimed at establishing equivalences between both versions of the tool. The proposed model defines six kinds of equivalences - conceptual, item, semantic, operational, measurement, and functional. This concept makes clear that the validation process is not to be concluded following an initial translation and reliability study, but rather is the final product of hard scientific work. We think that the cited papers of Bottino et al. (2001) and Paradela et al. (2009), along with a few other recently published methodological works, fail to fulfill the required criteria that would allow us to conclude that the task is finished.

Finally, in spite of the results presented by Aprahamian et al. (2011), the cumulative evidence of the last few decades is clear in pointing out that cultural level is heavily associated with cognitive performance, and that individuals with lower schooling have worse performance when compared with their higher schooled counterparts. Of course, there is no reason but one - bias - for a different behavior in the case of the CAMCOG. The hypothesis backing up the theory of cognitive reserve is that different kinds of lifelong stimuli are important factors involved in determining the cognitive capacity of the central nervous system. Therefore, besides schooling, participation in social, cultural, and labor activities are fundamental experiences which determine the overall capacity of the brain. Of course, there is no denying that individuals with higher socioeconomic status also have better access to information and 
services throughout their lives, as well as intellectual challenges that constitute important stimuli for improving their cognitive capacity. The sample in the Aprahamian et al. (2011) study was made up of 321 community-dwelling outpatients from a private geriatric institution. Although lacking full data on the sample's socioeconomic status, patients assisted at private clinics in Brazil are people with better economic conditions, since they have the means either to afford such services or to contribute to private health insurance policies. Probably, the better than average socioeconomic status was the variable chiefly responsible for a sample bias that selects a specific group of people with lower schooling and a better cognitive performance. Incidentally, the authors have suggested that "previous discrepancies associated with education bias have been found between the current sample ... and a sample derived from a public primary care unit in Rio de Janeiro" (Lourenço et al., 2008; Aprahamian et al., 2011), further reinforcing the point of view that the matter is not related to the instrument but rather to the specific characteristics of their sample.

Bottom line: sample and classification bias have determined the results and conclusions reported by Aprahamian et al. (2011), with which we vehemently disagree, i.e. that "the CAMCOG retains high accuracy when used in samples of elderly with limited education". Notwithstanding, we have to consider that these data validate the association between cognition and several other socioeconomic and critical cultural factors, always a major consideration in neuroscience projects.

\section{Conflict of interest}

None.

doi:10.1017/S1041610212000397

\section{Reply}

We thank you for the opportunity to respond to the letter written by Dr. Lourenço and Dr. Paradela, our colleagues from Rio de Janeiro. We understand scientific progress relies on lively debate and exchange of experiences; therefore, we also thank our colleagues for the opportunity to reflect upon our previous findings.

We identified three major issues in their letter and we respond to them in the items below.

\section{References}

Albert, M. S. et al. (2011). The diagnosis of mild cognitive impairment due to Alzheimer's disease: recommendations from the National Institute on Aging-Alzheimer's Association workgroups on diagnostic guidelines for Alzheimer's disease. Alzheimer's $\mathcal{E}$ Dementia, 7, 270 279.

Aprahamian, I., Martinelli, J. E., Cecato, J., Izbicki, R. and Yassuda, M. S. (2011). Can the CAMCOG be a good cognitive test for patients with Alzheimer's disease with low levels of education? International Psychogeriatrics, 23, 96-101.

Bottino, C. M. C., Stoppe, A., Jr., Scalco, A. Z., Ferreira, R. C. R., Hototian, S. R. and Scalco, M. Z. (2001). Validade e confiabilidade da versão brasileira do CAMDEX. Arquivos de Neuropsiquiatria, 59 (Suppl. 3), S20.

Herdman, M., Fox-Rushby, J. and Badia, X. (1998). A model of equivalence in the cultural adaptation of HRQoL instruments: the universalist approach. Quality Life Research, 7, 323-335.

Lourenço, R. A., Ribeiro-Filho, S. T., Moreira, I. F., Paradela, E. M. and Miranda, A. S. (2008). The Clock Drawing Test: performance among elderly with low educational level. Revista Brasileira de Psiquiatria, 30, 309-315.

Paradela, E. M., Lopes, C. S. and Lourenço, R. A. (2009). Reliability of the Brazilian version of the Cambridge Cognitive Examination Revised CAMCOG-R. Arquivos de Neuropsiquiatria, 67, 439-444.

\section{ROBERTO Alves LOURENÇO AND Emylucy Martins De Paiva Paradela Internal Medicine Department, Universidade do Estado do Rio de Janeiro, Rio de Janeiro, Brazil. Email: roberto.lourenco@globo.com}

1. $\mathrm{CDR}=0.5$ as Alzheimer's disease. It is well known that the Clinical Dementia Rating (CDR) was developed to stage cognitive impairment and that $\mathrm{CDR}=0.5$ may represent early stage $\mathrm{AD}$ or mild cognitive impairment (MCI). In our study, the 37 subjects with $\mathrm{CDR}=0.5$ presented with significant functional impairment and, therefore, were classified as early-stage AD (Mayeux, 2010).

2 . We agree with our colleagues that the validation of cognitive instruments developed under different cultural backgrounds is a complex process which requires several steps. Nonetheless, when we 